ACCEPTED MANUSCRIPT

\title{
The evaporation of the saline colloidal droplet and the deposition pattern
}

To cite this article before publication: Honghui Sun et al 2019 Chinese Phys. B in press https://doi.org/10.1088/1674-1056/ab5782

\section{Manuscript version: Accepted Manuscript}

Accepted Manuscript is "the version of the article accepted for publication including all changes made as a result of the peer review process, and which may also include the addition to the article by IOP Publishing of a header, an article ID, a cover sheet and/or an 'Accepted Manuscript' watermark, but excluding any other editing, typesetting or other changes made by IOP Publishing and/or its licensors"

This Accepted Manuscript is $\odot 2019$ Chinese Physical Society and IOP Publishing Ltd.

During the embargo period (the 12 month period from the publication of the Version of Record of this article), the Accepted Manuscript is fully protected by copyright and cannot be reused or reposted elsewhere.

As the Version of Record of this article is going to be / has been published on a subscription basis, this Accepted Manuscript is available for reuse under a CC BY-NC-ND 3.0 licence after the 12 month embargo period.

After the embargo period, everyone is permitted to use copy and redistribute this article for non-commercial purposes only, provided that they adhere to all the terms of the licence https://creativecommons.org/licences/by-nc-nd/3.0

Although reasonable endeavours have been taken to obtain all necessary permissions from third parties to include their copyrighted content within this article, their full citation and copyright line may not be present in this Accepted Manuscript version. Before using any content from this article, please refer to the Version of Record on IOPscience once published for full citation and copyright details, as permissions will likely be required. All third party content is fully copyright protected, unless specifically stated otherwise in the figure caption in the Version of Record.

View the article online for updates and enhancements. 
The evaporation of the saline colloidal droplet and the deposition pattern Honghui Sun(孙弘辉) $)^{13}$, Weibin Li(李伟斌) ${ }^{13}$,Wenjie Ji(纪文杰 $)^{13}$, Guoliang Dai(戴国亮) ${ }^{13}$, Yong

$$
\text { Huan(狍勇 } \left.)^{23} \text {, Yuren Wang(王育人 }\right)^{13} \text {, Ding Lan(蓝鼎) } *^{13}
$$

1 National Microgravity Laboratory, Institute of Mechanics, Chinese Academy of Sciences, 100190 Beijing, China 2 State Key Laboratory of Nonlinear Mechanics (LNM), Institute of Mechanics, Chinese Academy of Sciences, Beijing 100190, China

3 School of Engineering Sciences, University of Chinese Academy of Sciences, 100049 Beijing, China

*landing@imech.ac.cn

\begin{abstract}
The dynamic process of the evaporation and desiccation of sessile saline colloidal droplets, as well as their final deposition, is investigated. During the evaporation, the movement of the colloidal particles shows a strong dependence on the salt concentration and the droplet shape. The final deposition pattern indicates a weakened coffee-ring effect in this mixed droplet system. The microscopic observation reveals that, as evaporation proceeds, the particle motion trail is affected by the salt concentration of the droplet boundary. The Marangoni flows, which induced by surface tension gradients originating from the local evaporative peripheral salt enrichment, suppress the compensation flow towards the contact line of the droplet. The inhomogeneous density and concentration field induced by evaporation or crystallization could be the major reason for the various micro-flow. At last stage, the distribution and crystallization of $\mathrm{NaCl}$ are affected by the colloidal particles during the drying of the residual liquid film.
\end{abstract}

Keywords: saline droplet, evaporation, deposition pattern, disease diagnosis PACS:47.55.nb, 47.55.np, 45.70.Qj

\title{
1. Introduction
}

The evaporation of the sessile droplet is a ubiquitous phenomenon in nature, and in this process, there are many physical phenomena, such as convection, pinning and de-pinning of the contact line, spreading, wetting and de-wetting [1-4]. All of these phenomena have relationship with the micro-flows in the drying droplet, which controls the heat and mass transfer. Usually, the droplet is not a pure fluid but a complex fluid which contains solid particles or soluble multi-components. So, there will be some patterns remaining on the substrate. The evaporation of sessile droplets has significant value in research and application, such as nanomaterial fabrication [5], spray coating [6], DNA microarray design [7], and bio-liquid analysis [8]. For this reason, the microscopic mechanism of the evaporating droplets and control methods of dried patterns have attracted much attention in decades. For colloidal droplets, Deegan et al. firstly investigated the well-known "coffee-ring effect" [9]. They discovered that the faster evaporation rate at the pinned contact line is the main reason for the radial outward flow, which carries colloidal particles to form the ring-like stain at the edge. After their pioneering work, much effort has been made by 
researchers to regulate and control the coffee-ring effect for various practical applications. Man et al. proposed a theory for the multi-ring pattern of the deposits that are formed when droplets of the suspension were dried on a substrate [10]. Yunker et al. replaced the colloidal spheres in the droplets with ellipsoids and got a uniform deposition in which the coffee-ring effect is suppressed [11]. In 2001, BJ. Fisher specified the relationship between evaporative flux and direction of the radial flow inside the colloidal droplet [12].

In recent decades, the evaporation of sessile drops of biological fluid has been studied as a potential area of interest for disease diagnosis and they are trying to build the relationship between the drying patterns and health status of humans [13]. The patterns of dried blood serum from healthy people were significantly different, from the patients suffering from different diseases, such as paraproteinemia and lung cancer [14]. Most studies have been conducted on biological fluids and blood serum, but a few have focused on whole human blood. Rapis indicated that the morphologies of dried plasma patterns could be utilized for diagnosing metastatic carcinoma [15]. Brutin et al. found the dried drop patterns of whole blood from healthy individuals and those from patients suffering from anemia and hyperlipidemia are obviously different [16]. The researches mentioned above suggested dried blood pattern analysis could become an efficient tool for relatively easy and cheap disease diagnosis through further research effort.

However, biological liquid is a kind of complex fluids which, besides the cells, contains multi-components, such as biomolecules, inorganic electrolytes, and a majority of water etc. For the complicated interact of the various components in biological liquid droplet, it is very difficult to understand the pattern formation as the droplet dries. Even so, the researchers still do their best to find some clues and start with a simplified version of the biological fluid consisting of one or two biological components [17,18]. Studying the simpler, fluids may help us to understand the mechanisms of the pattern formation in more complex fluids. Shahidzadeh-Bonn et al. studied the crystallization process in the droplets with different dissolved salts on different substrates $[19,20]$. Their researches manifest that the patterns of the salt crystal stains are influenced by not only the transport properties in the liquid but also the interfacial properties of the different crystalline phases. For droplets of binary suspension, Chen and Mohamed studied the complex patterns formed after the evaporation of droplets containing protein and salt [17]. They proposed some theoretical explanations for the patterns obtained under different conditions. In addition, Zhang et al. investigated the evaporative pattern morphologies of pure DNA solution and DNA-colloid binary suspension [18]. They examined the influencing factors for the patterns with different morphologies and discussed the formation mechanism for the multi-ring pattern. Although it is not a real biological fluid, we still can tell us some information about the phenomena during the deposition of the biological.

Here, we observed the evaporation process of saline colloidal droplets in situ by confocal microscopy. We try to understand that the interaction between the soluble and insoluble substance during the droplet drying. We recorded the dynamic process of particles motion, salt crystallization, and droplet desiccation in real time with a CCD camera. By means of comparative analysis of the acquired images, we described the interplay of salt and colloidal particles, and we made some possible explanations. The consequences are meaningful for understanding the complex dried patterns of biological liquid droplets.

\section{Materials and methods}


The saline colloidal droplets are made up of polystyrene (PS) colloidal particles and sodium chloride $(\mathrm{NaCl})$ solution. The colloidal suspension of $10 \mathrm{wt} \%$ was purchased from Duke Scientific Corporation and the mean diameter of the colloidal spheres is $3.03 \mu \mathrm{m}$. The colloidal suspension and $\mathrm{NaCl}$ (AR, xilong scientific) was diluted with ultrapure water, which was obtained from Milli-Q Reference, to prepare the blended solution used in experiment. For comparison, various dispersions with different mass fraction of colloidal particles were prepared, and the $\mathrm{NaCl}$ was set at $0.9 \%$ to be close to the mass fraction of $\mathrm{NaCl}$ in plasma.

Micropipettes were used to fetch the drop and put it on the glass slides. The volume of each droplet is limited to about $1 \mu \mathrm{L}$. Since the physical properties of the slides are identical, the radiuses of the sessile droplets nearly are same. A confocal microscope (Nikon Ti) equipped with a digital camera was utilized to observe in-situ and record the evaporation process of droplets. The digital camera was set at a frame rate of $10 \mathrm{fps}$, which was enough for capturing the details. All the evaporation experiments were done in a conditioned environment with temperature of about $25^{\circ} \mathrm{C}$ and relative humidity of around $42 \%$.

\section{RESULTS \& DISCUSSION}

\subsection{Complete forming process of the deposition pattern in blended droplet}

The mass fraction of $\mathrm{NaCl}$ and PS colloidal particles are indicated by $\omega(\mathrm{NaCl})$ and $\omega(\mathrm{PS})$ respectively. For a saline colloidal droplet with $\omega(\mathrm{NaCl})$ equal to $0.9 \%$ and $\omega(\mathrm{PS})$ equal to $0.1 \%$, Fig. 1(a)-1(i) shows its whole drying process at a representative area on the edge of the droplet. According to the colloidal particles' motion status, we divide the whole process into three stages. The particles are moving stably towards the periphery of the circular droplet by the lateral flow at the beginning of evaporation. This period is defined as stage I, which corresponds to Fig. 1(a)-(c). Subsequently, the directional motion of the particles is disturbed by the emerging vortexes. Stage II is defined from this point till the moment when almost all the particles have adhered to the substrate and now the droplet gets very thin (Fig, 1(d)-1(e)). Finally, the particles move barely and the salt separates out until the droplet is totally dry (Fig. 1(f)-1(i)).

The colloidal particles migrate slowly and directionally to the three-phase contact line of the droplet due to a higher evaporation flux at the edge which is called compensation flow [21]. When the particles arrive at the contact line, they are confined by the meniscus and deposit on the substrate (see movie S1 in the Supplemental Material [22]). Within the stage I, a coffee ring forms [9,21], and this process lasts about two and a half minutes. At the stage II, several fast-rotating vortexes emerge in the droplet, causing more complex motions of colloidal particles in local areas (see movie S2 in the Supplemental Material [22]). At the same time, the coffee-ring at the border has a pinning effect for the liquid on triple-phase contact line [21], the radius of the droplet therefore barely reduces during this process. At the last stage of the drying process, the particles are adhered to the substrate due to the thin liquid film, and the particles are hard to move. Now, the droplet becomes a thin liquid film with a height close to the diameter of a colloidal particle, and the vortexes in the droplet gradually weaken until disappeared.

The salt crystallization and rupture of the liquid film occurs at the stage III (Fig. 1(f)-1(i)). From the full view of the deposition pattern, the big salt crystals are few and distribute sparsely, and some crystals look like dendritic crystals at the edge. When the salt crystals appear and grow up, the movement of the colloidal particles around the crystals are totally different. We can see the 
particles are pushed away from the crystals, and sometimes are drawn close to the crystals (see movie S3 in the Supplemental Material [22]). This is accounted for by the change of surface tension, which will be discussed in details later. As evaporation goes on, the liquid film begins to break when the residual solution is not enough to keep the integrity of the film. The ruptured liquid films collapse into plenty of small liquid patches, and there are several colloidal particles in them. At last, the residual liquid films evaporate so fast, that some incomplete salt crystals are left over. Figure1(j) gives a full view about the pattern.

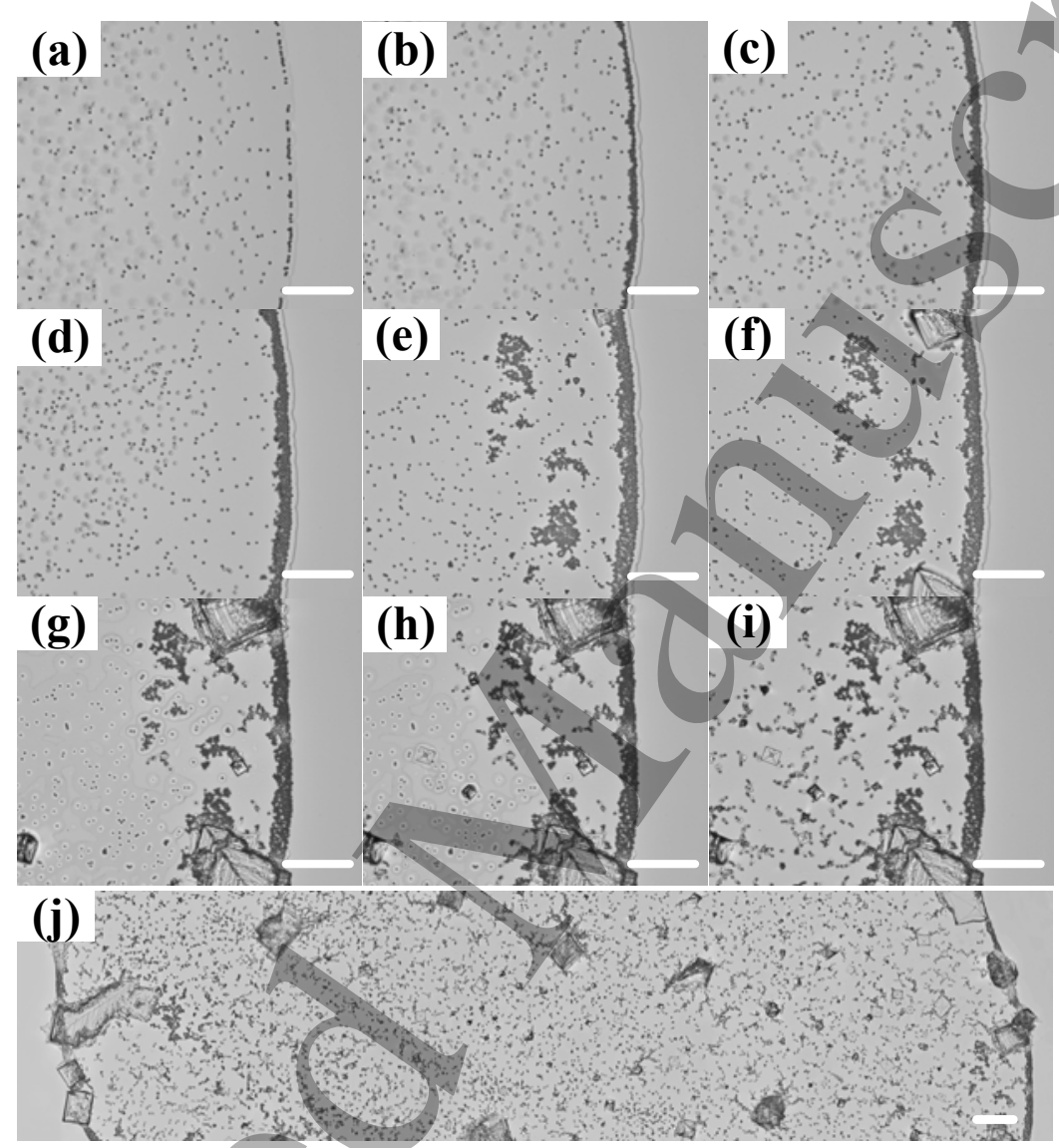

Fig. 1 (a)-(i) Drying process on the periphery of a blended droplet $(\omega(\mathrm{PS})=0.1 \%, \omega(\mathrm{NaCl})=0.9 \%)$ : (a)-(c) assembling of particles on the contact line of the droplet; (d) initiation of the vortex; (e) all particles are settled on the substrate; (f) crystallization of $\mathrm{NaCl}$; (g)-(h) rupture of the thin liquid film and small liquid pools formation; (i) total desiccation of the droplet; (j) full view of final transverse deposition. The scale bar is $100 \mu \mathrm{m}$.

\subsection{Effects of $\mathrm{NaCl}$ on the/deposition of the colloidal spheres}

The salt concentration can modify the aqueous surface tension [23], and the concentration will change during the evaporation. The local salt enrichment will induce the surface tension gradient, and the Marangoni flows will be induced which can influence the movement of the particles. At the same time, the crystalline of the salt will consume the salt in the water, and reduce the local salt concentration which will also lead to the surface tension gradient different from that mentioned before. The motion of the particles in the saline droplets is different from that in the 
pure colloidal droplets. We compared the movements of PS colloidal particles in these two kinds of droplets that have the same concentration of PS, to analyze how the micro-flow is affected by salt.

\subsubsection{Retarding the radical speed of particles}

At the beginning, the colloidal particles move to the edge of the droplet for the compensation flow, and the mechanism has been clarified by Deegan [9]. Different from the pure liquid, the salt concentration would increase as the saline solution drying, and we try to understand the influence of the varying concentration for the movement of the particles by checking the mean radial speeds of the particles. First, we acquired the image sequence of the area near the border of the droplet in stage I, and the frame rate was $10 \mathrm{fps}$. A series of frames were picked out at regular intervals of 60 seconds, beginning with the first frame. Then we picked out several typical particles from each frame. The selected particles were traced within dozens of serial frames to determine their flowing distance before they deposited at the contact line. We would calculate their mean radial speeds at different time periods which could give the information about the micro-flow, and compare them to find influence of concentration change on the particle motion. The whole process is illustrated in Fig. 2.

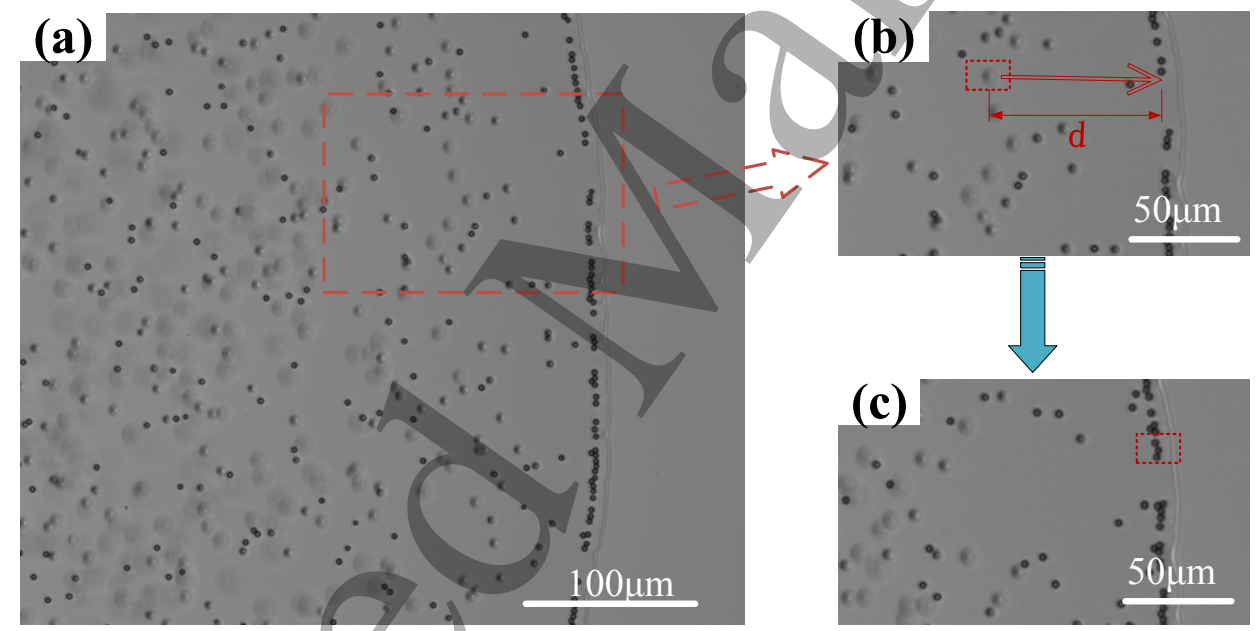

Fig. 2 Estimating the radial speed of certain particles. At a certain point in time, a typical particle was chosen and traced in a short duration around the point. The local area around it in (a) is magnified to see clearly in (b) and (c). The red frame in (b) shows the initial position of the particle, and (c) shows the final position. The displacement of the particle is denoted by $\mathrm{d}$ in (b). The time is defined by counting the frames within the interval between (b) and (c).

In stage $\mathrm{I}$, there is a stable radial flow towards the edge of the droplet. Since the flow is roughly axisymmetric for circular droplets [9], the calculated speed of the particles in a limited area near the edge is representative. It shows the intensity of compensation flow in the whole droplet. We compared the speed trends of the particles in the saline droplet and in pure water droplet (shown in Fig.3). The concentration of colloidal particles is kept the same in these two experimental systems. In the case of saline colloidal droplet, represented by the blue triangles in Fig.3, the initial speed was around $6.5 \mu \mathrm{m} / \mathrm{s}$. The value decreased to $5.3 \mu \mathrm{m} / \mathrm{s}$ in 60 seconds, while 
there was not significant change in speed after another 60 seconds. In the next 30 seconds, the speed slowed down further to $4.4 \mu \mathrm{m} / \mathrm{s}$. By contrast, in the case of pure colloidal droplet, represented by the red squares in Fig.3, the speed increased from $3.7 \mu \mathrm{m} / \mathrm{s}$ at beginning to $18.6 \mu \mathrm{m} / \mathrm{s}$ seven minutes later. But the rate of increase was not uniform. In the first 4 minutes, the speed increased by about $3 \mu \mathrm{m} / \mathrm{s}$. While in the next 3 minutes, it increased by about $12 \mu \mathrm{m} / \mathrm{s}$.

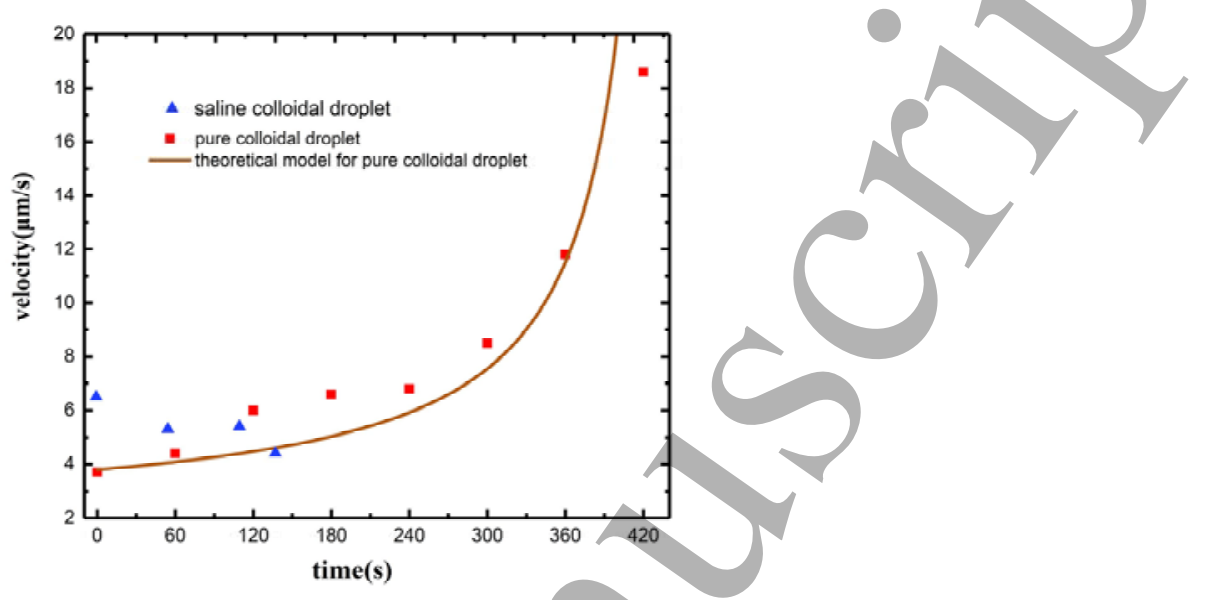

Fig. 3 Plot of the radial particle speed versus time. The particles that are measured are about $100 \mu \mathrm{m}$ away from the contact line. Blue triangles represent particles in blended droplet $(\omega(\mathrm{NaCl})=0.9 \%, \omega(\mathrm{PS})=0.1 \%)$, which in pure colloidal droplet $(\omega(\mathrm{PS})=0.1 \%)$ are represented by red squares.

Marín et al. described the variation of particles' radical speed inside an evaporating colloidal droplet quantitatively [24], which will be discussed below by comparing with the experimental results in two kinds of droplet systems. In this model, the height-averaged radial speed $\bar{u}$ close to the contact line is expressed as

$$
\bar{u}(r, t)=\frac{D^{*}}{\theta(t)} \frac{1}{\sqrt{R(R-r)}}
$$

In equation (1), $D^{*}=2 \sqrt{2} D_{v a} \Delta c /(\pi \rho)$ represents the effect of flow driven by evaporation, with $D_{v a}$ the diffusion constant for vapor in air, $\Delta c$ the vapor concentration difference between the drop surface and the surroundings, $\rho$ the liquid density. In addition, $R$ is the contact radius of the droplet, which is determined for a certain droplet on a substrate; $r$ is the distance from drop center; $\theta$ is the contact angle as a function of time t. For an evaporating colloidal droplet whose contact line is pinned, the evaporation follows the constant contact area mode [25], and contact angle decreases over time $\mathrm{t}$ in this mode. In order to make further analysis, the theoretical model actually gives the expression for $\frac{d \theta}{d t}$ by global mass conservation:

$$
\frac{d \theta}{d t}=-\frac{16 D_{v a} \Delta c}{\pi R^{2} \rho}
$$

For a pure water droplet evaporating in a certain ambient environment, the parameters at the right side of equation (2) are constants. Thus equation (2) is equal to a constant, independent of time. The time integration of equation (2) is: 


$$
\theta(\mathrm{t})=\frac{16 D_{v a} \Delta c}{\pi R^{2} \rho}\left(t_{e}-t\right)
$$

where $t_{e}$ is the total life time of the droplet. Plugging (3) into (1), we obtain:

$$
\bar{u}(r, t)=\frac{\sqrt{2} R}{8\left(t_{e}-t\right)} \sqrt{\frac{R}{R-r}}
$$

$\mathrm{R}$ and $t_{e}$ is determined from experiment. Since the particles we measured are at a constant distance from the contact line, R-r is also determined. The theoretical curve of $\bar{u}$ varying with $\mathrm{t}$ is plotted in Fig. 3. The original theoretical curve is actually a little lower than the experimental data points. For the convenience of comparing, we have shifted the theoretical curve upward about two units to approach the experimental data points. This does not affect the comparison results and conclusion significantly, considering that the relative variation trend of speed is our main focus.

Obviously, the variation trend of speed in experimental pure colloidal droplet matches up well enough with the theoretical model. However, the speed in saline colloidal droplet has a distinct tendency, which implies the dissolved salt plays an important role in retarding the outward flow. The theoretical model also indicates this. As the evaporation goes on in the saline colloidal droplet, the concentration of salt increases, which results in the growth/of $\rho$. With $\rho$ as a function of $t$, the integration of equation (2) will not be equation (3) any longer, and $\theta$ will not be inversely proportional to $\rho$. When equation (3) is inserted into equation (1), the parameter $\rho$ cannot be eliminated. Consequently, the final expression of $\bar{u}$ also involves density $\rho$. Hence, the variation trend of $\bar{u}$ over time is influenced by the growth of $\rho$ in blended droplet, which is definitely distinct from the curve of equation (4).

In fact, when the density of blended droplet, $\rho$ increases over time, factor $\mathrm{D}^{*}$ will decrease. This can be derived from the relational expression of $\mathrm{D}^{*}$ and $\rho$. The particle speed $\bar{u}$ is on a decreasing trend in this situation, which means the flow driven by evaporation is weakened by the increasing of density. On the other hand, the particle speed $\bar{u}$ has a tendency to increase with the deceasing of droplet contact angle $\theta$. This is determined by conservation of mass. Consequently, the change of particle speed $\bar{u}$ depends on how the ratio of $\mathrm{D}^{*}$ to $\theta$ changes over time. When the influence on the particle speed made by the weakening of evaporation-driven flow with increase in density is enough to counteract that made by the decreasing of contact angle, the particles will definitely be retarded. This is the situation in blended droplet, where the effect of density increase weakening evaporation-driven flow becomes dominant and finally $\bar{u}$ decreases over time.

\subsubsection{Emergence of vortexes}

In our experiment, another remarkable phenomenon is that there is an emergence of vortexes in the saline droplet in stage 2 (see movie S2 in the Supplemental Material [22]). The particle speeds in the vortex is much/greater than other region in the droplet. The vortex exits in limited areas, and it only affects the particles in it or it, which is different from the global ring currents in droplet that is mentioned in some papers. The vortex forms when most of the particles in the droplet are moving towards the edge, and its direction and scale keep evolving after its formation. Generally, one vortex can be seen in the field of view. But sometimes, symmetrical vortexes are observed. Compared with this, there is not vortex from beginning to end in pure colloidal droplet. Therefore, the dissolved salt in the droplet is supposed to be a key factor for the formation of vortex. 
The effect of salt on vortex formation could come from two aspects. On the one hand, the inhomogeneous evaporation of different parts in the droplet brings about a concentration gradient of $\mathrm{NaCl}$, which leads to a density gradient as the concentration of solution is related to its density. If the density gradient is large enough, it will cause a buoyancy-driven flow [26], which may evolve into a vortex afterwards. On the other hand, the evaporation rate is faster near the edge of the droplet surface, while it is slower at the center. This uneven local evaporation rate will also lead to a concentration gradient of $\mathrm{NaCl}$ along the droplet surface. Generally speaking, the surface tension of a saline droplet increases with the rising of salt concentration [27], thus a surface tension gradient is generated. This will also cause flows on the surface and in the droplet when the gradient is sufficiently large, which is called the flow driven by Marangoni effect/The vortex can also derive from this kind of flow.

In theory, the two different formation mechanisms of vortex should exist simultaneously. Quantitative comparisons of the intensity of the two factors can be made through discussion of two dimensionless numbers: the Rayleigh number Ra which characterizes the strength of the buoyancy effect, and the Marangoni number Ma which characterizes the strength of the Marangoni effect. The parameters that affect the value of these two dimensionless numbers remain to be determined by further experiments.

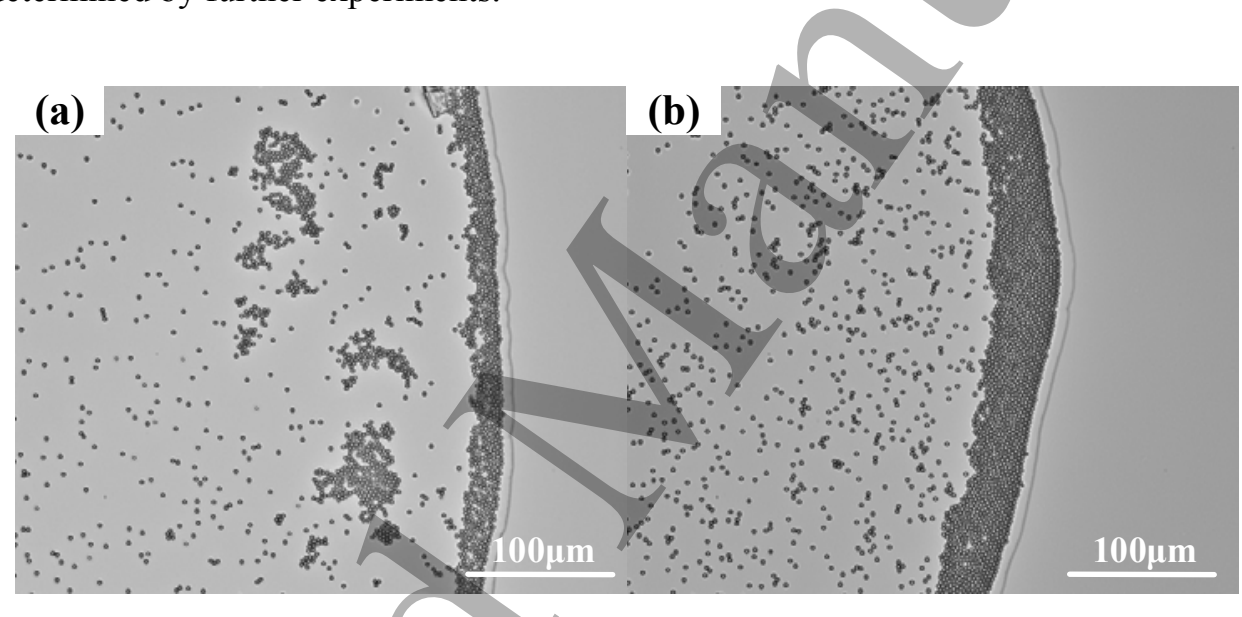

Fig. 4 Deposition morphology of colloidal particles on the periphery of: (a) saline colloidal droplet $(\omega(\mathrm{PS})=0.1 \%$, $\omega(\mathrm{NaCl})=0.9 \%)$, (b)pure colloidal droplet.

The dissolved salt in the blended droplet has retarded the radial migration of particles in stage 1 , and has led to the formation of vortex in stage 2, which also disturbed the movement and deposition of colloidal particles. These factors have come together to affect the morphology of the ring deposit significantly at the edge of droplet. The coffee ring morphologies at the boundary of blended droplets and pure colloidal droplets are compared in Figure 4. It can be seen clearly that, in the ring deposit of pure colloidal droplet, the configuration of the particles is more compact, while in the blended droplet it is relatively looser. In addition, the width of coffee ring deposit in pure colloidal droplet is nearly twice that in blended droplet. Such results indicate that the addition of salt can affect the micro-flow within the droplet during the evaporation, thereby changing the deposition morphology of the colloidal particles.

\subsubsection{Influence of growing $\mathrm{NaCl}$ crystal on the surrounding particles}


When the salt in the droplet nucleates and crystallizes, the motion of the colloidal particles around the growing crystals of $\mathrm{NaCl}$ are affected more significantly. But if the crystallization happens after all particles are settled, the effect is not conspicuous as the particles are bound to the substrate and restricted to move, which is the case of our above experiments.

In order to observe the effect of salt crystallization, we also carried out experiments with $20 \%$ salt solution droplets. In this case, the solution reaches saturation before all colloidal particles are settled. When the crystal nucleuses form and start to grow, the motion trend of the particles in the region adjacent to the grains of salt is changed in different ways (see movie $\mathrm{S} 3$ in the Supplemental Material [22]). The colloidal particles near the superficial layer or in it are pushed away from the grains. The repulsion effect of the grains has an action range. At a distance which is several times of the length of a crystalline grain from the center of this grain, the effect weakens to a low level. The colloidal particles are barely pushed further away at that distance. This is possibly because a local surface tension gradient which is caused by the gradient of salt concentration near the grains. The regions close to the grains are of a lower concentration as the salt in the droplet is consumed when the $\mathrm{NaCl}$ crystal appear and grow up. As discussed by Sghaier et al., surface tension of a saline droplet increases with the salt concentration $[27,28]$. The liquid surface tension near the $\mathrm{NaCl}$ crystal is smaller (shown by Fig.5).

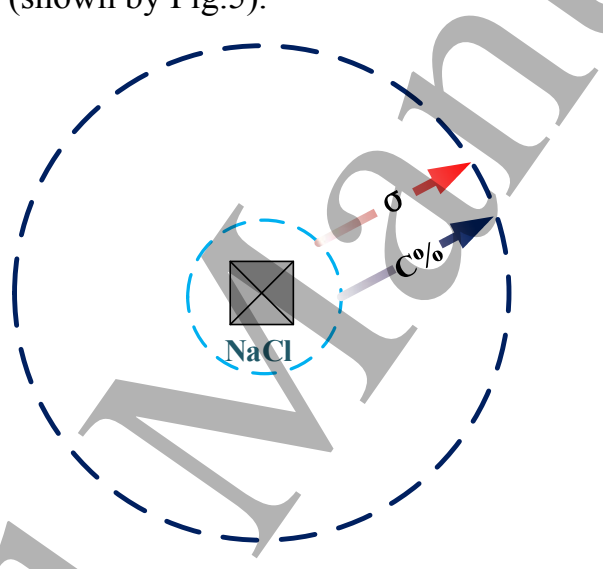

Fig. 5 The influence of the $\mathrm{NaCl}$ crystal to the salt concentration and the surface tension. $\sigma$ - the surface tension, $\mathrm{C} \%$ -the salt concentration.

However, the particles under the superficial layer seem to be affected by the grains of salt in different ways. When the crystals are growing, a considerable number of colloidal particles under the superficial layer dash quickly towards the grains. Some of them hit the surface of the grains and are bounced off, while some others get into the gap between the grains and the substrate [see movie S3 in the Supplemental Material [22]]. The reason for the unusual flow towards the grains of salt is inferred to be a compensate flux caused by the outward flow near the superficial layer.

\subsection{Effects of PS colloidal particles on NaCl crystals}

The deposition patterns of saline colloidal droplet and pure saline droplet are compared to analyze whether the PS colloidal particles have an effect on the crystallization of $\mathrm{NaCl}$. The mass fraction of $\mathrm{NaCl}$ in the droplets is prepared as $0.9 \%$. The volume of the droplets is kept the same as the previous experiments. 
We first measured the radii of the region of dried deposition with scattered $\mathrm{NaCl}$ crystals. The radius of the dried blended droplet, which could be obtained from Fig. 1(j), is about $1.27 \mathrm{~mm}$. By contrast, the radius of the dried pure saline droplet (see Fig. 6) is around $1.20 \mathrm{~mm}$. The shrinkage of diameter in pure saline droplet indicates that its contact line recedes more during evaporation. Further observation reveals the reason for the differences.

It is notable that the coffee ring formed by the deposited colloidal particles in the blended droplet has a pinning effect on the boundary contact line of the droplet due to the hydrophilia of PS colloidal spheres. When the droplet is evaporating, the water cannot separate from the coffee ring easily even though the receding contact angle generates an inward driving force to the contact line. As a result, the radius of the droplet is stable before the solution reaches saturation. And finally the salt crystallizes in the whole region of droplet, which is an approximate circular defined by the coffee ring.

By contrast, the radius of a pure saline droplet keeps decreasing during evaporation as the receding of the contact line is not impeded. When the solution reaches saturation and starts to crystallize, the radius of the droplet has reduced a lot. Thus the eventual crystalline region is smaller. In a word, the range of salt crystallization in saline colloidal droplet is affected by the colloidal particles through pinning the boundary contact line of the droplet.

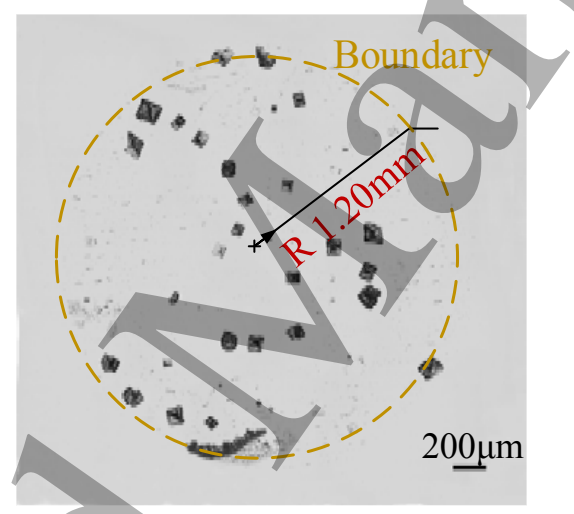

Fig. 6 Morphology of dried deposits in pure saline droplet $(\omega(\mathrm{NaCl})=0.9 \%)$ and its radius of deposition.

Moreover, we recorded the sequential images of stage 3 at the center of the blended droplet in smaller scale (see Fig. 7). It could be found that the colloidal particles also work as a factor to control the formation of the salt crystal grains. At sage 3, the droplet has shrunk to a thin liquid film. Almost all the particles are settled on the substrate. At this time, an initial crystal have come out (as pointed by the red arrow in Fig. 7(b)). Soon the liquid film ruptures at a moment (as shown by the yellow dashed circle in Fig. 7(c)). Subsequently, the gaps expands gradually in the intervals of the settled particles. The liquid film breaks into a mass of patches eventually (some examples are marked with red circles in Fig. 7(e)). Every patch contains several colloidal particles, due to the hydrophilia of the particles. 


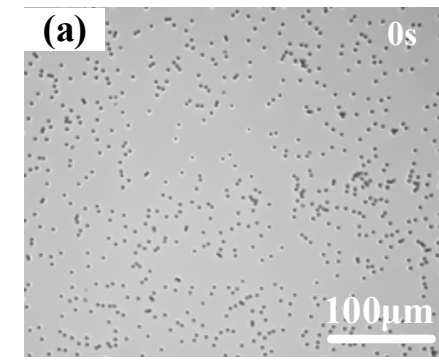

(d)

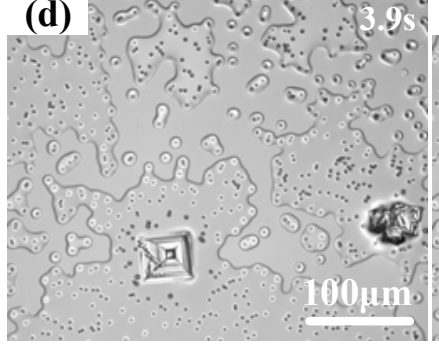

(b)

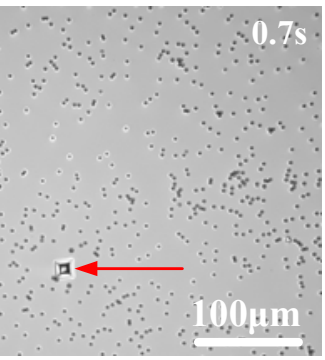

(e)

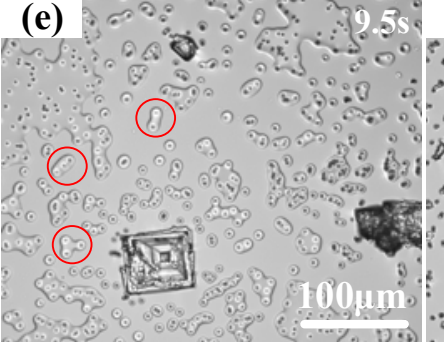

(c)

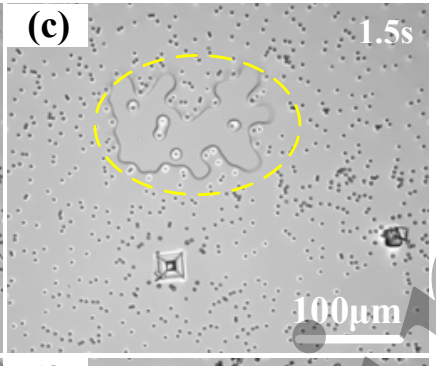

(f)

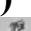

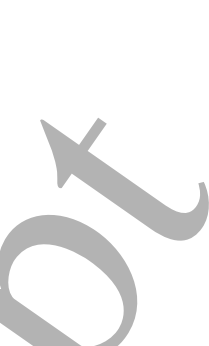
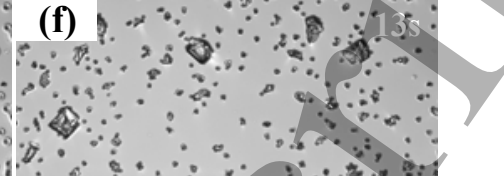

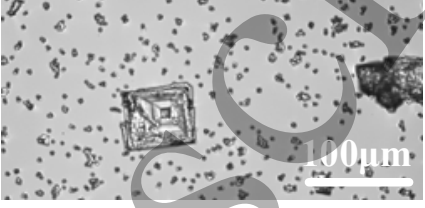

Fig. 7 Last stage of drying in chronological order: (a) Local area of the liquid film continues getting thinner. (b) The first wave of crystals appear. (c)Rupture of the liquid film. (d) Further rupture and formation of liquid patches. (e) The liquid patches add and the pointed crystal in Fig.7(b) grows up (f) Totally dried deposits.

Due to the unique role of colloidal particles, the quantity of emergent crystalline grains in the blended droplet depends on how many patches the residual liquid film is divided into. In comparison, in pure saline droplet, the residualliquid film does not break up into so many patches during evaporation because of the lack of colloidal particles. Therefore, the salt mainly separates out on a few initial crystal grains (as shown in Fig. 6). This determines that there are more scattered salt crystal grains in the final deposition patterns of saline colloidal droplet. In addition, the average size of the grains also has decreased. Because the salt in the blended droplet is separated into more grains, which leads to less quantity of salt in each one. Accordingly, the colloidal particles have a significant influence on $\mathrm{NaCl}$ crystals.

\section{Conclusion}

We study the different stages of the drying sessile saline colloidal droplets, including the final pattern formation. By comparison and analysis, it is found that the micro-flow in blended droplets is significant affected due to the existence of salt. The evaporation-driven radial compensation flow is slowed down. In addition, there are commonly rapid-rotating vortices appearing during the forming stage of coffee-ring. And an outward flow around the growing $\mathrm{NaCl}$ crystals is also observed. The gradient of density field and concentration field are considered to be the main reason for the change of micro-flow. On the other hand, it is confirmed that the distribution range and grain size of $\mathrm{NaCl}$ crystals are affected by colloidal particles through changing the dewetting process of the residual liquid film. Under the interplay of salt and colloidal particles, the deposition pattern shows obvious differences between the blended droplet and mono-component droplet. The consequences imply that the deposition facies of some biological liquid could be analyzed to acquire important information about its physiological characteristics. 


\section{Acknowledgment}

We gratefully acknowledge financial support from the National Natural Science Foundation of China (Grant Nos 11472275 and U1738118), CAS Key Technology Talent Program, and the Strategic Priority Research Program of the Chinese Academy of Sciences (Grant No. XDB22040301).

\section{References}

[1] Lubarda V A 2012 Soft Matter 810288.

[2] Weon B M and Je J H 2013 Phys Rev Lett 110.

[3] Nikolov A D, Wasan D T, Chengara A, Koczo K, Policello G A and Kolossvary I 2002 Adv Colloid Interfac 96325.

[4] Nikolov A and Wasan D 2014 Adv Colloid Interfac 206207

[5] Li W B, Lan D and Wang Y R 2017 Phys Rev E 95.

[6] Yu Y, Zhu H, Frantz J M, Reding M E, Chan K C and Ozkan H E 2009 Biosyst Eng 104324.

[7] Smalyukh I I, Zribi O V, Butler J C, Lavrentovich O D and Wong G C L 2006 Phys Rev Lett 96.

[8] Shatokhina S N, Shabalin V N, Buzoverya M E and Punin V T 2004 The Scientific World Journal 4657.

[9] Deegan R D, Bakajin O, Dupont T F, Huber G, Nagel S R and Witten T A 1997 Nature 389 827.

[10] Wu M M, Man X K and Doi M 2018 Langmuir 349572.

[11] Yunker P J, Still T, Lohr M A and Yodh A G 2011 Nature 476308.

[12] Fischer B J 2002 Langmuir 1860.

[13] Sefiane K 2014 Adv Colloid Interfac 206372.

[14] Yakhno T A, Yakhno V G, Sanin A G, Sanina O A, Pelyushenko A S, Egorova N A, Terentiev I G, Smetanina S V, Korochkina O V and Yashukova E V 2005 Ieee Eng Med Biol 2496.

[15] Rapis E 2002 Tech Phys+ 47510.

[16] Brutin D, Sobac B, Loquet B and Sampol J 2011 J Fluid Mech 66785.

[17] Chen G F and Mohamed G J 2010 Eur Phys J E 3319.

[18] Zhang L, Maheshwari S, Chang H C and Zhu Y X 2008 Langmuir 243911.

[19] Shahidzadeh-Bonn N, Rafai S, Bonn D and Wegdam G 2008 Langmuir 248599.

[20] Shahidzadeh N, Schut M F L, Desarnaud J, Prat M and Bonn D 2015 Sci Rep-Uk 5.

[21] Deegan R D, Bakajin O, Dupont T F, Huber G, Nagel S R and Witten T A 2000 Phys Rev E 62756.

[22] the Supplemental Materials for the experimental movies. 
[23] Soulie V, Karpitschka S, Lequien F, Prene P, Zemb T, Moehwald H and Riegler H 2015 Phys Chem Chem Phys 1722296.

[24] Marin A G, Gelderblom H, Lohse D and Snoeijer J H 2011 Phys Rev Lett 107.

[25] Picknett R G and Bexon R 1977 J Colloid Interf Sci 61336.

[26] Kang K H, Lim H C, Lee H W and Lee S J 2013 Phys Fluids 25.

[27] Sghaier N, Prat M and Ben Nasrallah S 2006 Chem Eng J 12247.

[28] Weast R C 1980 CRC Handbook of Chemistry and Physics. 60th Edition (CRC Press).

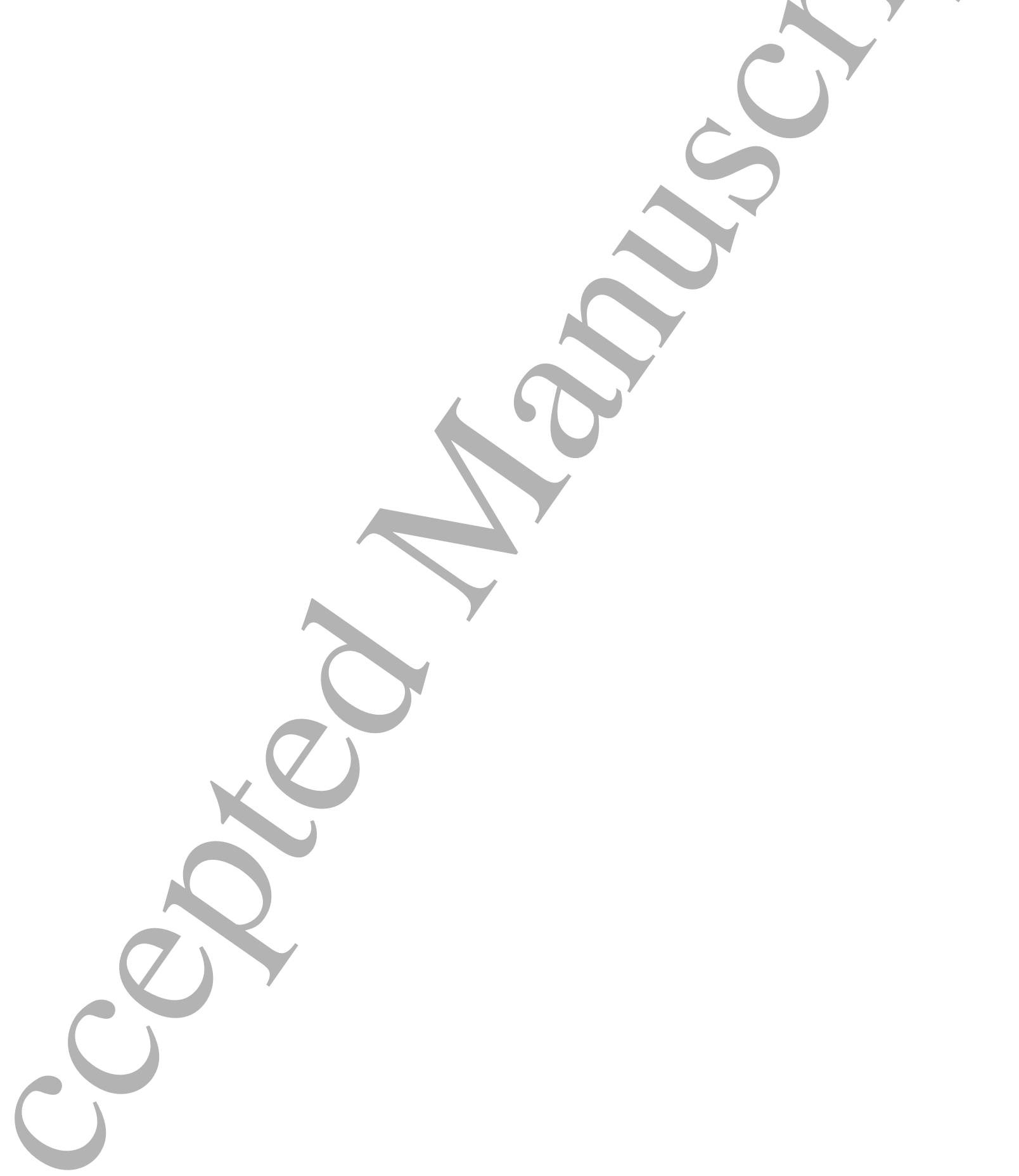

